\title{
PERTURBATIONS OF A ROTATING BLACK HOLE
}

\author{
SAUL A. TEUKOLSKY \\ California Institute of Technology, Pasadena, Calif., U.S.A.
}

\begin{abstract}
Decoupled, separable wave equations describing neutrino, electromagnetic and gravitational perturbations have been derived (Teukolsky, 1972, 1973). A number of applications have been made (Press and Teukolsky, 1973; Starobinsky and Churilov, 1973).
\end{abstract}

\section{References}

Press, W. H. and Teukolsky, S. A.: 1973, Astrophys. J. 185, 649.

Starobinsky, A. A. and Churilov, S. M.: 1973, Zh. E.T.F. 65, 3.

Teukolsky, S. A.: 1972, Phy's. Rev. Letters 29, 1114.

Teukolsky, S. A.: 1973, Astrophys. J. 185, 635. 